\title{
Description of processes passing at finite space and time intervals in the framework of QFT
}

\author{
Vadim Egorov ${ }^{1,2, *}$ and Igor Volobuev ${ }^{2, * *}$ \\ ${ }^{1}$ Faculty of Physics, M.V. Lomonosov Moscow State University \\ ${ }^{2}$ Skobeltsyn Institute of Nuclear Physics, M.V. Lomonosov Moscow State University
}

\begin{abstract}
We consider a new approach to the description in the framework of QFT of processes passing at finite space and time intervals. The formalism is based on the Feynman diagram technique in the coordinate representation, in which the rules of passing to the momentum representation are modified in accordance with the experimental setup of neutrino oscillation experiments. In effect, only the propagators of particles in the momentum representation are modified, while all the other standard Feynman rules in the momentum representation remain the same. Since the initial and final particle states are described by plane waves, the approach does not need the use of wave packets, which greatly simplifies the calculations of amplitudes. Taking as examples the processes of displaced pion decay, neutral kaon and neutrino oscillations we show that the approach under consideration correctly reproduces the known standard results.
\end{abstract}

\section{Introduction}

The standard perturbative S-matrix formalism and Feynman diagram technique allow one to describe a huge number of various processes of elementary particle interactions, and the results of the theoretical description are in an excellent agreement with the experimental data in the vast majority of cases. This primarily applies to the processes of elementary particle scattering. However, there is a number of phenomena that cannot be described within the framework of the standard perturbation theory: these are, in particular, the phenomena of neutral meson and neutrino oscillations that occur at macroscopic space and time intervals. For these phenomena one uses either a quantum mechanical description in terms of plane waves, or a field-theoretical description in terms of wave packet.

The quantum mechanical description in terms of plane waves has been put forward in paper [1] to explain oscillations of neutral kaons and subsequently generalized to the case of neutrino oscillations in papers $[2,3]$. A detailed discussion of the current status of this approach can be found in papers [4-7]. In this approach it is assumed that the particle states produced in the strong or weak interactions are not particle mass eigenstates, but the states with certain quantum numbers, - the strangeness and lepton flavor. The evolution of these states in time is found by expanding them in mass eigenstates and is defined by the free Hamiltonians. As a result, over time, the states with a definite strangeness or lepton flavor

\footnotetext{
*e-mail: egorov@theory.sinp.msu.ru

**e-mail: volobuev@theory.sinp.msu.ru
} 
become superpositions of states with different values of strangeness or with different lepton flavors. The use of the states with a definite strangeness or lepton flavor for describing the neutrino interactions and decays of neutral kaons leads to the well-known formulas for oscillations of neutrinos and neutral kaons, which are in a good agreement with the experimental data.

However, long ago it was noted that the quantum-mechanical description in terms of plane waves was inconsistent, because energy-momentum conservation was violated in the production processes of states that did not have a definite mass [8-12]. As a result, a new quantum field-theoretical approach to the description of neutrino oscillations was put forward in paper [8], which, according to the provisions of quantum field theory, suggests that the neutrino mass eigenstates produced in the weak interactions are off the mass shell and described by Feynman propagators, and neutrino oscillations occur due to interference of the amplitudes of all three virtual neutrinos in the intermediate state. There are no problems with conservation of energy-momentum in this approach, however, it is poorly compatible with the standard S-matrix perturbation theory, which is not adapted for describing the processes passing at finite space or time interval. To account for finite distances in experiments on neutrino oscillations, in the S-matrix perturbation theory it is necessary to use localized wave packets, which greatly complicate the calculations.

In the present paper we put forward a method of quantum field-theoretical description of processes passing at finite space and time intervals, which combines the simplicity of the standard quantum-mechanical approach through the use of plane waves and the rigor of the field-theoretical approach using wave packets. The proposed formalism is based on the Feynman diagram technique in the coordinate representation, supplemented by modified rules for the transition to the momentum representation. Calculations in its framework use the standard Feynman diagram technique in the momentum representation, in which a modified propagator is used instead of the Feynman propagator.

This approach has been proposed in papers [13-17]. In the present paper we show that the application of this approach to describing the processes of displaced unstable particle decay, neutral kaon and neutrino oscillations correctly reproduces the known results.

\section{Displaced pion decay}

To explain the ideas of the approach, we consider the process of pion photoproduction and decay at a macroscopic distance $L$ from the source into a muon and a neutrino mass eigenstate. The latter means that we work in the minimal extension of the Standard Model by the right neutrino singlets. The diagram of the process looks like

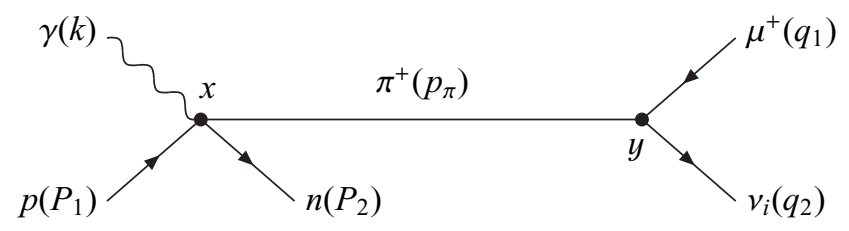

Here the intermediate pion is a virtual particle and is described by the Feynman propagator in the coordinate space. We assume that the points $x$ and $y$ are separated by a fixed macroscopic distance $L$. Since only the antimuon is detected experimentally, the probability of this process with $i$-th neutrino mass eigenstate in the final state must be summed over $i=1,2,3$. As it is customary in the Feynman diagram technique, we assume that the initial and final particles are described by plane waves, i.e. have definite momenta. Therefore, the virtual pion also has 
a definite momentum. Let us denote the 4-momenta of the particles as it is shown in the diagram, namely, the photon has momentum $k$, the proton $P_{1}$, the neutrino $P_{2}$, the intermediate virtual pion $p_{\pi}$, the antimuon $q_{1}$, and the $i$-th neutrino mass eigenstate $q_{2}$.

The amplitude in the coordinate representation corresponding to diagram (1) can be easily written out using the standard Feynman rules in the coordinate representation, formulated, for example, in the textbook [18]. For the transition to the momentum representation, according to the prescription of the standard S-matrix theory ([18], §24), it would be necessary to integrate this amplitude with respect to the coordinates $x$ and $y$ of the interaction points over Minkowski space, which would give the corresponding matrix element of the S-matrix. However, such integration would lead to the loss of information about the space-time interval between the production and decay events, while the experimental setup suggests that distance between the production and decay points remains constant. In order to generalize the standard perturbative formalism to the case of processes occurring at fixed distances, it is necessary to modify the integration so that it takes into account the fixed distance between the production and decay points of the pion. This can be done by introducing the delta function $\delta(\vec{n}(\vec{y}-\vec{x})-L)$ into the integrand, where $\vec{n}$ is a unit vector, directed from the source to the detector. In doing so, we fix the distance $L$ between the interaction points along the line connecting the source and detector, and only then integrate the amplitude with respect to the coordinates $x$ and $y$ over Minkowski space. Thus, just like in the standard S-matrix formalism, we consider the process occurring throughout space-time, but the distance between the production and decay points in the direction from the source to the detector (i.e. along the momentum of the pion beam) is now fixed by the delta function. Of course, this is at odds with the standard S-matrix formalism. But it is worth recalling that the diagram technique in the coordinate representation was developed by R. Feynman [19, 20] regardless of the S-matrix theory. Thus, Feynman diagrams in the coordinate representation have meaning beyond the framework of this theory, and we can integrate the amplitude with respect to $x$ and $y$ in any way depending on the considered physical problem. The introduction of the additional delta function is formally equivalent to the replacement of the standard Feynman propagator $D^{\mathrm{c}}(y-x)$ of the scalar particle in the coordinate representation by $D^{\mathrm{c}}(y-x) \delta(\vec{n}(\vec{y}-\vec{x})-L)$. The Fourier transform of the last expression gives us the so called distance-dependent propagator of scalar particles in the momentum representation.

However, it turns out that a more convenient for calculations and physically more fundamental object is a time-dependent propagator. It is obtained by introducing the delta function $\delta\left(y^{0}-x^{0}-T\right)$ into the integrand of the amplitude of the process, which fixes the time interval $T$ between the production point of the pion and its decay point. Fixing the time interval $T$ between these events instead of the distance $L$ is a technique that is convenient for the development of a consistent formalism. Of course, actually it is the distance between the pion source and the detector that is fixed in the experiments, but for a particle beam with the same momentum, this is equivalent to fixing the time interval between the production and decay events in accordance with the formula $T=L p^{0} /|\vec{p}|$.

Thus, the introduction of a time-lapse delta function is equivalent to replacing the Feynman propagator $D^{\mathrm{c}}(y-x)$ by $D^{\mathrm{c}}(y-x) \delta\left(y^{0}-x^{0}-T\right)$. By definition, the Fourier transform of this expression gives us the time-dependent propagator of the scalar particle in the momentum representation:

$$
D^{\mathrm{c}}(p, T)=\int \mathrm{d}^{4} x e^{i p x} D^{\mathrm{c}}(x) \delta\left(x^{0}-T\right) .
$$


Let us forget for a moment that the pion is an unstable particle and neglect its width. Then this integral can be evaluated exactly by contour integration, the result being given by $[13,14]$

$$
D^{\mathrm{c}}(p, T)=i \frac{1}{2 \sqrt{\left(p^{0}\right)^{2}+m_{\pi}^{2}-p^{2}-i \varepsilon}} e^{i\left(p^{0}-\sqrt{\left(p^{0}\right)^{2}+m_{\pi}^{2}-p^{2}}\right) T}
$$

In paper [9] it was rigorously proved that the particles described by the Feynman propagators in the coordinate space and propagating at macroscopic distances (or over macroscopic time intervals) are almost on the mass shell, i.e. the ratio $\left|p^{2}-m_{\pi}^{2}\right| /\left(p^{0}\right)^{2} \ll 1$ for such particles. Thus, for large macroscopic time intervals $T$ we can expand the time-dependent propagator in this ration, keeping it only in the exponential. This gives

$$
D^{\mathrm{c}}(p, T) \simeq i \frac{1}{2 p^{0}} e^{-i \frac{m_{\pi}^{2}-p^{2}}{2 p^{0}} T} .
$$

Taking the pion width $\Gamma$ into account slightly modifies this expression, so that it becomes

$$
D^{\mathrm{c}}(p, T) \simeq i \frac{1}{2 p^{0}} e^{-i \frac{m_{\pi}^{2}-p^{2}-i m_{\pi} \Gamma}{2 p^{0}} T} .
$$

Now we are ready to proceed to the calculation of the probability of the process corresponding to diagram (1). Using time dependent propagator (5), we write out the amplitude of the process in the momentum representation in the case, where the time interval $y^{0}-x^{0}$ between the production and decay events is fixed and equals $T$ :

$$
M^{(i)}=i \frac{G_{\mathrm{F}}}{2 \sqrt{2} p_{\pi}^{0}} \cos \theta f_{\pi} m_{(\mu)} U_{2 i}^{*} e^{-i \frac{m_{\pi}^{2}-p_{\pi}^{2}-i m_{\pi} \Gamma}{2 p_{\pi}^{0}} T} \bar{v}_{i}\left(q_{2}\right)\left(1-\gamma^{5}\right) v\left(q_{1}\right) M_{\mathrm{P}}\left(k, P_{1}, p_{\pi}, P_{2}\right) .
$$

Here $\theta$ is the Cabibbo angle, $f_{\pi}$ is the decay constant of the pion of the dimension of mass, $m_{(\mu)}$ is the muon mass, $U_{i k}$ denotes the Pontecorvo-Maki-Nakagawa-Sakata (PMNS) mixing matrix, and we have already applied the 4-momentum conservation condition at the pion decay vertex $([21], \S 5) . M_{\mathrm{P}}\left(k, P_{1}, p_{\pi}, P_{2}\right)$ denotes the photoproduction amplitude of the $\pi$ meson that depends on four form factors; it is a quite bulky expression that we do not really need to care about, so here it is not written out explicitly. The index $(i)$ on the left side of the equality means that the $i$-th neutrino mass eigenstate is produced in the final state.

Let us find the square of the amplitude modulus averaged over the polarizations of the initial particles and summed over the polarizations of the final particles (averaging and summation operations are denoted by the anglular brackets). Since the amplitude proper, due to the simple structure of the scalar propagator, is the product of the amplitudes of the pion production and decay subprocesses, the squared amplitude also factorizes:

$$
\begin{aligned}
\left\langle\left|M^{(i)}\right|^{2}\right\rangle & =\left\langle\left|M_{1}\right|^{2}\right\rangle\left\langle\left|M_{2}^{(i)}\right|^{2}\right\rangle \frac{1}{4\left(p_{\pi}^{0}\right)^{2}} e^{-\frac{m_{\pi} \Gamma}{p_{\pi}^{0}} T}, \\
\left\langle\left|M_{1}\right|^{2}\right\rangle & =\frac{1}{4}\left|M_{\mathrm{P}}\left(k, P_{1}, p_{\pi}, P_{2}\right)\right|^{2}, \\
\left\langle\left|M_{2}^{(i)}\right|^{2}\right\rangle & =4 G_{\mathrm{F}}^{2} \cos ^{2} \theta f_{\pi}^{2} m_{(\mu)}^{2}\left|U_{2 i}\right|^{2}\left(q_{1} q_{2}\right),
\end{aligned}
$$

where $\left\langle\left|M_{1}\right|^{2}\right\rangle$ is the squared amplitude of the $\pi^{+}$-meson photoproduction, $\left\langle\left|M_{2}^{(i)}\right|^{2}\right\rangle$ is the squared amplitude of the $\pi^{+}$-meson decay into an antimuon and the $i$-th neutrino mass eigenstate in the approximation of the zero masses of the latter. Since in amplitude (6) there is only 
one term, in expression (7) there is no contribution from the oscillating part of the propagator. There remains only the decay exponential factor.

We have come to finding the probability of the process under consideration, when the time interval between the points $x$ and $y$ is equal to $T$. We recall that the amplitude in the momentum representation corresponding to diagram (1) contains, along with the expression in formula (6), the delta function of energy-momentum conservation. Thus, following the prescription of the standard perturbative approach, for finding the probability of the process we should multiply the squared amplitude (7) by the delta function of energy-momentum conservation $(2 \pi)^{4} \delta\left(k+P_{1}-P_{2}-q_{1}-q_{2}\right)$ and integrate the result over the phase space for the final particles. However, since $p_{\pi}$ is defined only by the law of 4-momentum conservation at the production vertex, $p_{\pi}=k+P_{1}-P_{2}$, such integration would result in a variation in the direction of the momentum $\vec{p}_{\pi}$ of virtual pions. This is contrary to the conditions of the experiment, where the virtual $\pi$-mesons propagate in the direction defined by the mutual location of the source and detector. Thus, we need to calculate the differential probability of the process for $p_{\pi}$, fixed by the experimental situation.

We introduce a 4-momentum $p$ of the intermediate pion: $p^{2}=m_{\pi}^{2}$ and the vector $\vec{p}$ satisfies the condition of energy-momentum conservation at the production vertex and, according to the experimental setup, is directed from the source to the detector. In general, choosing one value $\vec{p}$ of the pion momentum is an approximation applicable, when the distance between the source and the detector is much larger than their own sizes. The required differential probability can be obtained by multiplying the squared amplitude modulus (7), besides the delta function of energy-momentum conservation, also by the delta function $2 \pi \delta\left(p_{\pi}-p\right)$, which fixes the momentum of the intermediate pion, or, equivalently, by replacing $p_{\pi}$ with $p$ everywhere in formula (7) and multiplying it by the additional delta function $2 \pi \delta\left(k+P_{1}-P_{2}-p\right)$. Only after this substitution it is allowed to integrate over the phase space of the final particles in accordance with the standard rules for calculating probabilities, namely the neutron, the antimuon and the $i$-th neutrino mass eigenstate. The extra factor $2 \pi$ appears due to the averaging over the directions of the momenta of the virtual pions, which is necessary because of the non-zero sizes of the source and detector.

Due to the factorization of the squared amplitude the differential probability also factorizes. Now that the momentum of the virtual pions is fixed and is equal to $p$, we can go from the time interval $T$ to the distance traveled by the pion before the decay $L$ in accordance with the formula $T=L p^{0} /|\vec{p}|$. This substitution is legitimate, because the pions are almost on the mass shell and $|\vec{p}| / p^{0}$ can be considered as the speed of a pion with very high accuracy. Summing the resulting probability over the type $i$ of the final neutrinos, which is reduced to summing the squares of the matrix elements of the PMNS matrix in formula (9) and gives unity as a result, we arrive at the following expression:

$$
\begin{aligned}
\frac{\mathrm{d}^{3} W}{\mathrm{~d}^{3} p}= & \left.\frac{1}{2 k^{0} 2 P_{1}^{0}} \int \frac{\mathrm{d}^{3} P_{2}}{(2 \pi)^{3} 2 P_{2}^{0}} \frac{\mathrm{d}^{3} q_{1}}{(2 \pi)^{3} 2 q_{1}^{0}} \frac{\mathrm{d}^{3} q_{2}}{(2 \pi)^{3} 2 q_{2}^{0}} \sum_{i=1}^{3}\left\langle\left|M^{(i)}\right|^{2}\right\rangle\right|_{\substack{p_{\pi}=p \\
T=L p^{0} /|\vec{p}|}} \times \\
& \times(2 \pi)^{4} \delta\left(k+P_{1}-P_{2}-q_{1}-q_{2}\right) 2 \pi \delta\left(k+P_{1}-P_{2}-p\right)=\frac{\mathrm{d}^{3} W_{1}}{\mathrm{~d}^{3} p} W_{2} e^{-\frac{m_{\pi} \Gamma}{|\vec{p}|} L},
\end{aligned}
$$

where $\mathrm{d}^{3} W_{1} / \mathrm{d}^{3} p$ is the differential probability of photoproduction of $\pi^{+}$-meson with momentum $\vec{p}$ and $W_{2}$ is the probability of the $\pi^{+}$-meson decay into an antimuon and a neutrino mass eigenstate, which, in the limit of zero neutrino masses, coincides with the standard probability of the $\pi^{+}$-meson decay into an antimuon and a massless muon neutrino. Thus, we got that the differential probability of the whole process is the product of the differential probability of the production of a $\pi^{+}$-meson with a certain momentum, the probability of its decay, and 
the standard exponential factor, describing the survival probability of the pion on its way to the detector.

Finally, we see that the experimental situation fixes only the direction of the momentum of the intermediate pion, but not its length. Therefore, in order to find the probability of the process it is necessary to integrate differential probability (10) multiplied by $|\vec{p}|^{2}$ with respect to $|\vec{p}|$ over the admissible values of the latter. In the case under consideration the production process has a two-particle final state, which means that the lengths of the pion and neutron momenta are already fixed by energy-momentum conservation (only their directions can vary). As a result, the differential probability of the production process is singular, and the singularity is eliminated by integration. The final result for the probability of the whole process looks like

$$
\frac{\mathrm{d} W}{\mathrm{~d} \Omega}=\int \frac{\mathrm{d}^{3} W}{\mathrm{~d}^{3} p}|\vec{p}|^{2} \mathrm{~d}|\vec{p}|=\left.\frac{\mathrm{d} W_{1}}{\mathrm{~d} \Omega} W_{2}\right|_{|\vec{p}|=|\vec{p}|^{*}} e^{-\frac{m_{\pi} \Gamma}{|\vec{p}|^{*}} L},
$$

where $|\vec{p}|^{*}$ is the root of the equation $k^{0}+P_{1}^{0}-\left.P_{2}^{0}\right|_{\vec{P}_{2}=\vec{k}+\vec{P}_{1}-\vec{p}}-p^{0}=0$ for $|\vec{p}|$. The resulting expression (11) is in a full agreement with the result expected from the physical considerations.

\section{Neutral Kaon oscillations}

Now we apply the developed methods to describe the oscillations of neutral kaons. To do this, we consider a process, in which $K^{0}$-mesons are produced, when a proton target is irradiated by a beam of $\pi^{-}$-mesons, $\pi^{-}+p \rightarrow K^{0}+\Lambda^{0}$. Then they propagate over a macroscopic time $T$ that we assume to be fixed and finally decay into $\pi^{+} \pi^{-}$-pairs.

Since $K_{\mathrm{S}}^{0}$ and $K_{\mathrm{L}}^{0}$ are produced in the process of strong interaction, we cannot definitely isolate the subprocesses of the $K_{\mathrm{S}}^{0}, K_{\mathrm{L}}^{0}$ formation at the quark level. However we can assume that at the point $x$, either a virtual $K_{\mathrm{S}}^{0}$ or a virtual $K_{\mathrm{L}}^{0}$ is produced and decays into $\pi^{+} \pi^{-}$-pair at the point $y$. Thus, the process can be represented by the following diagram:

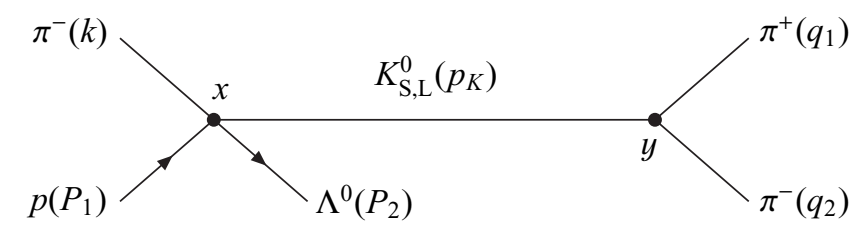

As elsewhere in this paper, we assume that the states of the initial particles are described by plane waves, i.e. the initial and, consequently, the final particles and intermediate kaons have definite momenta. Let us denote them as shown in the diagram: the 4-momentum of the initial $\pi^{-}$-meson there is $k$, that of the proton $-P_{1}$, that of the $\Lambda$-hyperon $-P_{2}$, that of the intermediate $K_{\mathrm{S}}^{0}$ (or $K_{\mathrm{L}}^{0}$ ) - $p_{K}$, that of the $\pi^{+}$-meson $-q_{1}$, and that of the $\pi^{-}$-meson $-q_{2}$.

The amplitudes of the complete processes of a $\pi^{+} \pi^{-}$-pair production by $K_{\mathrm{S}}^{0}$ and $K_{\mathrm{L}}^{0}$ differ in the amplitudes of kaon production, in the propagators and in the amplitudes of the decay into pions. We denote by $P_{\mathrm{S}}\left(k, P_{1}, p_{K}, P_{2}\right)$ the production amplitude of $K_{\mathrm{S}}^{0}$ and by $A_{+-, \mathrm{S}}$ its decay amplitude to $\pi^{+} \pi^{-}$; and, similarly, we denote by $P_{\mathrm{L}}\left(k, P_{1}, p_{K}, P_{2}\right)$ the production amplitude of $K_{\mathrm{L}}^{0}$ and by $A_{+-, \mathrm{L}}$ its decay amplitude to $\pi^{+} \pi^{-}$, all the amplitudes taken in the momentum representation. Since the energy release in the decay $K_{\mathrm{S}, \mathrm{L}}^{0} \rightarrow \pi^{+}+\pi^{-}$is small (slightly more than $200 \mathrm{MeV}$ ), we can neglect the composite nature of these scalar particles and assume that the decay amplitudes are independent of the momenta, i.e. are constants.

Next we use the time-dependent propagator of an unstable scalar particle in the momentum representation (5) to write down the amplitude corresponding to diagram (12) with fixed 
time interval $y^{0}-x^{0}=T$ :

$$
\begin{aligned}
M=\frac{i}{2 p_{K}^{0}} e^{-i \frac{m_{\mathrm{S}}^{2}-p_{K}^{2}-i m_{\mathrm{S}} \Gamma_{\mathrm{S}}}{2 p_{K}^{0}} T} P_{\mathrm{S}}\left(k, P_{1}, p_{K}, P_{2}\right) A_{+-, \mathrm{S}}+ \\
+\frac{i}{2 p_{K}^{0}} e^{-i \frac{m_{\mathrm{L}}^{2}-p_{K}^{2}-i m_{\mathrm{L}} \Gamma_{\mathrm{L}}}{2 p_{K}^{0}} T} P_{\mathrm{L}}\left(k, P_{1}, p_{K}, P_{2}\right) A_{+-, \mathrm{L}},
\end{aligned}
$$

where $m_{\mathrm{S}}\left(m_{\mathrm{L}}\right)$ and $\Gamma_{\mathrm{S}}\left(\Gamma_{\mathrm{L}}\right)$ are, respectively, the mass and width of the state $K_{\mathrm{S}}^{0}\left(K_{\mathrm{L}}^{0}\right)$. Introducing the standard notation $\eta_{+-}=A_{+-, \mathrm{L}} / A_{+-, \mathrm{S}}$, we can rewrite the amplitude in the form:

$$
M=\frac{i}{2 p_{K}^{0}} P_{\mathrm{S}}\left(k, P_{1}, p_{K}, P_{2}\right) A_{+-, \mathrm{S}}\left[e^{-i \frac{m_{\mathrm{S}}^{2}-p_{K}^{2}-i m_{\mathrm{S}} \Gamma_{\mathrm{S}}}{2 p_{K}^{0}} T}+\frac{P_{\mathrm{L}}\left(k, P_{1}, p_{K}, P_{2}\right)}{P_{\mathrm{S}}\left(k, P_{1}, p_{K}, P_{2}\right)} \eta_{+-} e^{-i \frac{m_{\mathrm{L}}^{2}-p_{K}^{2}-i m_{\mathrm{L}} \Gamma_{\mathrm{L}}}{2 p_{K}^{0}} T}\right]
$$

It is convenient to isolate the phases of $\eta_{+-}$and $P_{\mathrm{L}}\left(k, P_{1}, p_{K}, P_{2}\right) / P_{\mathrm{S}}\left(k, P_{1}, p_{K}, P_{2}\right)$, by choosing the following parameterization:

$$
\eta_{+-}=\left|\eta_{+-}\right| e^{i \phi_{+-}}, \quad \frac{P_{\mathrm{L}}\left(k, P_{1}, p_{K}, P_{2}\right)}{P_{\mathrm{S}}\left(k, P_{1}, p_{K}, P_{2}\right)}=\left|\frac{P_{\mathrm{L}}\left(k, P_{1}, p_{K}, P_{2}\right)}{P_{\mathrm{S}}\left(k, P_{1}, p_{K}, P_{2}\right)}\right| e^{i \phi_{P}\left(k, P_{1}, p_{K}, P_{2}\right)} .
$$

The squared modulus of the process amplitude averaged over the polarizations the initial particles and summed over the polarizations of the final particles takes the form

$$
\begin{aligned}
\left\langle|M|^{2}\right\rangle= & \frac{1}{8\left(p_{K}^{0}\right)^{2}}\left|P_{\mathrm{S}}\left(k, P_{1}, p_{K}, P_{2}\right)\right|^{2}\left|A_{+-, \mathrm{S}}\right|^{2} \times \\
& \times\left[e^{-\frac{m_{\mathrm{S}} \Gamma_{\mathrm{S}}}{p_{K}^{0}} T}+\left|\frac{P_{\mathrm{L}}\left(k, P_{1}, p_{K}, P_{2}\right)}{P_{\mathrm{S}}\left(k, P_{1}, p_{K}, P_{2}\right)}\right|^{2}\left|\eta_{+-}\right|^{2} e^{-\frac{m_{\mathrm{L}} \Gamma_{\mathrm{L}}}{p_{K}^{0}} T}+\right. \\
& +2\left|\frac{P_{\mathrm{L}}\left(k, P_{1}, p_{K}, P_{2}\right)}{P_{\mathrm{S}}\left(k, P_{1}, p_{K}, P_{2}\right)}\right|\left|\eta_{+-}\right| e^{-\frac{m_{\mathrm{S}} \Gamma_{\mathrm{S}}+m_{\mathrm{L}} \Gamma_{\mathrm{L}}}{2 p_{K}^{0}} T} \times \\
& \left.\quad \times \cos \left(\frac{m_{\mathrm{L}}^{2}-m_{\mathrm{S}}^{2}}{2 p_{K}^{0}} T-\phi_{P}\left(k, P_{1}, p_{K}, P_{2}\right)-\phi_{+-}\right)\right] .
\end{aligned}
$$

Let us calculate the probability of the process under consideration. We denote the 4momentum of the $K$-mesons by $p: p^{2}=\left(m_{\mathrm{S}}+m_{\mathrm{L}}\right)^{2} / 4, p=k+P_{1}-P_{2}$, where, in accordance with the experimental situation, the momentum $\vec{p}$ is directed from the source (the target irradiated by a $\pi^{-}$-meson beam) to the detector. Following the previously formulated recipe, for finding the differential probability of the process we must multiply the expression (16) by the delta function of energy-momentum conservation $(2 \pi)^{4} \delta\left(k+P_{1}-P_{2}-q_{1}-q_{2}\right)$, replace there everywhere $p_{K}$ by $p$, multiply by the delta function $2 \pi \delta\left(k+P_{1}-P_{2}-p\right)$, fixing the momentum of the intermediate $K_{\mathrm{S}}^{0}$ - and $K_{\mathrm{L}}^{0}$-mesons, and then to integrate with respect to the momenta of the final particles according to the usual rules of finding the probabilities. We recall that the initial particles (negative pion and proton) have definite momenta; when we, following the experimental setup, also fix the 4-momentum of the intermediate kaons with the help of the corresponding delta function, the momentum $P_{2}$ of the $\Lambda$-hyperon in the final state is also fixed. From now on the squared moduli of the production amplitudes $P_{\mathrm{S}}$ and $P_{\mathrm{L}}$ do not depend on the momenta of final particles, as well as their ratio $P_{\mathrm{L}} / P_{\mathrm{S}}$. In fact we get that the total squared amplitude (16) does not depend on the momenta of the final particles and may be taken out from under the integral sign.

The formulas describing neutral kaon oscillations are usually written in terms of the proper time. In the considered case it should be approximately defined as the average of 
the proper times of $K_{\mathrm{S}}^{0}$ and $K_{\mathrm{L}}^{0}$,

$$
t_{\mathrm{p}}=\frac{m_{\mathrm{S}}+m_{\mathrm{L}}}{2 p^{0}} T
$$

With this in mind we obtain the differential probability of the process in the form:

$$
\begin{aligned}
\frac{\mathrm{d}^{3} W}{\mathrm{~d}^{3} p}=\frac{1}{2 k^{0} 2 P_{1}^{0}} \int & \left.\frac{\mathrm{d}^{3} P_{2}}{(2 \pi)^{3} 2 P_{2}^{0}} \frac{\mathrm{d}^{3} q_{1}}{(2 \pi)^{3} 2 q_{1}^{0}} \frac{\mathrm{d}^{3} q_{2}}{(2 \pi)^{3} 2 q_{2}^{0}}\left\langle|M|^{2}\right\rangle\right|_{\substack{p_{K}=p \\
T=\frac{2 p^{0}}{m_{\mathrm{S}}+m_{\mathrm{L}}} t_{\mathrm{p}}}} \times \\
& \times(2 \pi)^{4} \delta\left(k+P_{1}-P_{2}-q_{1}-q_{2}\right) 2 \pi \delta\left(k+P_{1}-P_{2}-p\right)= \\
= & \frac{\mathrm{d}^{3} W_{1}}{\mathrm{~d}^{3} p} W_{2}\left[e^{-\frac{2 m_{\mathrm{S}} \Gamma_{\mathrm{S}}}{m_{\mathrm{S}}+m_{\mathrm{L}}}}+\left|\frac{P_{\mathrm{L}}}{P_{\mathrm{S}}}\right|^{2}\left|\eta_{+-}\right|^{2} e^{-\frac{2 m_{\mathrm{L}} \Gamma_{\mathrm{L}}}{m_{\mathrm{S}}+m_{\mathrm{L}}} t_{\mathrm{p}}}+\right. \\
& \left.+2\left|\frac{P_{\mathrm{L}}}{P_{\mathrm{S}}}\right|\left|\eta_{+-}\right| e^{-\frac{m_{\mathrm{S}} \Gamma_{\mathrm{S}}+m_{\mathrm{L}} \Gamma_{\mathrm{L}}}{m_{\mathrm{S}}+m_{\mathrm{L}}} t_{\mathrm{p}}} \cos \left(\left(m_{\mathrm{L}}-m_{\mathrm{S}}\right) t_{\mathrm{p}}-\phi_{P}-\phi_{+-}\right)\right],
\end{aligned}
$$

where

$$
\frac{\mathrm{d}^{3} W_{1}}{\mathrm{~d}^{3} p}=\frac{1}{2 k^{0} 2 P_{1}^{0}} \frac{1}{(2 \pi)^{3} 2 p^{0}} \int \frac{\mathrm{d}^{3} P_{2}}{(2 \pi)^{3} 2 P_{2}^{0}}\left\langle\left|P_{\mathrm{S}}\right|^{2}\right\rangle(2 \pi)^{4} \delta\left(k+P_{1}-P_{2}-p\right)
$$

is the differential probability of the production of a $K_{\mathrm{S}}^{0}$-meson with momentum $\vec{p}$ in the reaction $\pi^{-}+p \rightarrow K^{0}+\Lambda^{0}$ and

$$
W_{2}=\frac{1}{2 p^{0}} \int \frac{\mathrm{d}^{3} q_{1}}{(2 \pi)^{3} 2 q_{1}^{0}} \frac{\mathrm{d}^{3} q_{2}}{(2 \pi)^{3} 2 q_{2}^{0}}\left|A_{+-, \mathrm{S}}\right|^{2}(2 \pi)^{4} \delta\left(p-q_{1}-q_{2}\right)
$$

is the decay probability of $K_{\mathrm{S}}^{0}$ into a pair $\pi^{+} \pi^{-}$.

Finally, we integrate (18) multiplied by $|\vec{p}|^{2}$ with respect to $|\vec{p}|$. Since there are two particles in the final state of the $K^{0}$ production process, this integration again removes the delta function that remains in $\mathrm{d}^{3} W_{1} / \mathrm{d}^{3} p$. The final result can be presented as follows:

$$
\begin{aligned}
\frac{\mathrm{d} W}{\mathrm{~d} \Omega}=\int \frac{\mathrm{d}^{3} W}{\mathrm{~d}^{3} p}|\vec{p}|^{2} d|\vec{p}|= & \left.\frac{\mathrm{d} W_{1}}{\mathrm{~d} \Omega} W_{2}\right|_{|\vec{p}|=|\vec{p}|^{*}}\left[e^{-\frac{2 m_{\mathrm{S}} \Gamma_{\mathrm{S}}}{m_{\mathrm{S}} m_{\mathrm{L}}} t_{p}}+\left|\frac{P_{\mathrm{L}}}{P_{\mathrm{S}}}\right|^{2}\left|\eta_{+-}\right|^{2} e^{-\frac{2 m_{\mathrm{L}} \Gamma_{\mathrm{L}}}{m_{\mathrm{S}} m_{\mathrm{L}}} t_{p}}+\right. \\
& \left.+2\left|\frac{P_{\mathrm{L}}}{P_{\mathrm{S}}}\right|\left|\eta_{+-}\right| e^{-\frac{m_{\mathrm{S}} \Gamma_{\mathrm{S}}+m_{\mathrm{L}} \Gamma_{\mathrm{L}}}{m_{\mathrm{S}}+m_{\mathrm{L}}} t_{p}} \cos \left(\left(m_{\mathrm{L}}-m_{\mathrm{S}}\right) t_{p}-\phi_{P}-\phi_{+-}\right)\right]\left.\right|_{|\vec{p}|=|\vec{p}|^{*}},
\end{aligned}
$$

where $|\vec{p}|^{*}$ is the root of the equation $k^{0}+P_{1}^{0}-\left.P_{2}^{0}\right|_{\vec{P}_{2}=\vec{k}+\vec{P}_{1}-\vec{p}}-p^{0}=0$ with respect to $|\vec{p}|$. We have again obtained that the total probability of the process has the form of the product of the production probability of a neutral kaon with the definite momentum, the probability of its decay and the factor having both oscillating and damping terms, the latter describing the decay. For $P_{\mathrm{L}}=P_{\mathrm{S}}$ and $P_{\mathrm{L}}=-P_{\mathrm{S}}$ this expression exactly coincides with the one presented in [4], $\$ 4.3$ if one neglects the difference between $m_{\mathrm{L}}$ and $m_{\mathrm{S}}$ in all the exponentials. Formula (21) can also be rewritten as

$$
\begin{aligned}
\frac{\mathrm{d} W}{\mathrm{~d} \Omega}=\left.\frac{\mathrm{d} W_{1}}{\mathrm{~d} \Omega} W_{2}\right|_{|\vec{p}|=|\vec{p}|^{*}} & {\left[\left(e^{-\frac{m_{\mathrm{S}} \Gamma_{\mathrm{S}}}{m_{\mathrm{S}}+m_{\mathrm{L}}} t_{\mathrm{p}}}+\left|\frac{P_{\mathrm{L}}}{P_{\mathrm{S}}}\right|\left|\eta_{+-}\right| e^{-\frac{m_{\mathrm{L}} \Gamma_{\mathrm{L}}}{m_{\mathrm{S}} m_{\mathrm{L}}} t_{\mathrm{p}}}\right)^{2}-\right.} \\
& \left.-4\left|\frac{P_{\mathrm{L}}}{P_{\mathrm{S}}}\right|\left|\eta_{+-}\right| e^{-\frac{m_{\mathrm{S}} \Gamma_{\mathrm{S}}+m_{\mathrm{L}} \Gamma_{\mathrm{L}}}{m_{\mathrm{S}}+m_{\mathrm{L}}} t_{\mathrm{p}}} \sin ^{2}\left(\frac{m_{\mathrm{L}}-m_{\mathrm{S}}}{2} t_{\mathrm{p}}-\frac{\phi_{P}+\phi_{+-}}{2}\right)\right]\left.\right|_{|\vec{p}|=|\vec{p}|^{*}} .
\end{aligned}
$$

Thus, we have again reproduced the results of the standard approach without the use of wave packets and states with definite strangeness $K^{0}, \bar{K}^{0}$. 


\section{Neutrino oscillations}

Now we will discuss neutrino oscillation processes, which turns out to be easier, because massive neutrinos can be regarded as stable particles [22]. These oscillations are usually understood as a neutrino transition from one neutrino flavor state into a different neutrino flavor state depending on the distance traveled [5-7]. This is related to the standard quantummechanical description of this phenomenon, which is based on the notion of neutrino flavor states, i.e. the states with a definite lepton flavor. It is assumed that it is the flavor states that are produced in the weak interaction. They can be considered as superpositions of the neutrino mass eigenstates, and the evolution of the flavor states in time is the cause of neutrino oscillations.

This description of neutrino oscillations is simple and physically transparent. However, as we have mentioned in the Introduction, this description violates energy-momentum conservation. An alternative field-theoretical description proposed in paper [8] and developed in papers $[9,10]$ explains neutrino oscillations by interference of the amplitudes of processes with different virtual neutrino mass eigenstates. In the framework of this description there is no problem with energy-momentum conservation, but the calculations of amplitudes of processes are very difficult because of the need to use wave packets. Below we show that neutrino oscillations can be consistently described in the framework of quantum field theory using only plane waves, which greatly simplifies the calculations. At the same time in the developed approach the law of energy-momentum conservation is also fulfilled.

As we have already noted, we work within the framework of the minimal SM extension by the right neutrino singlets. Below we consider the case, where neutrinos are produced and detected in the weak charged current interaction with nuclei. The weak charged current interaction Lagrangian of leptons has the form:

$$
L_{\mathrm{cc}}=-\frac{g}{2 \sqrt{2}}\left(\sum_{i, k=1}^{3} \bar{l}_{i} \gamma^{\mu}\left(1-\gamma^{5}\right) U_{i k} v_{k} W_{\mu}^{-}+\text {H.c. }\right),
$$

where $l_{i}$ denotes the charged lepton field of the $i$-th generation, $v_{k}$ is the field of a neutrino mass eigenstate, and $U_{i k}$ is the PMNS matrix. Due to this structure of the Lagrangian, any process involving the production of a neutrino at one point and its registration at another point can be represented in the lowest order of perturbation theory by the following diagram:

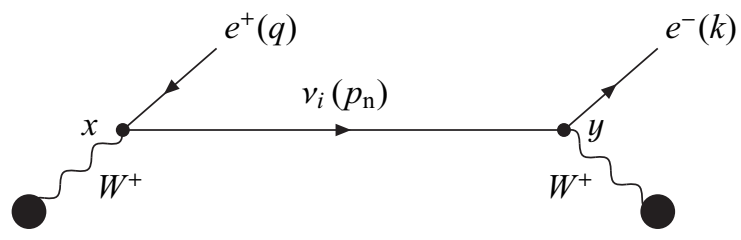

All the three intermediate virtual neutrino mass eigentates $v_{i}$ contribute to the amplitude of the process, therefore, the amplitude of the process corresponding to this diagram should be summed over all three of these states, $i=1,2,3$. We assume that the virtual $W$ bosons are produced and absorbed in the charged current interactions with the nuclei of the following form: nucleus ${ }_{Z_{1}}^{A_{1}} \mathrm{X}$, which we will call nucleus 1 , emits a $W^{+}$boson and turns into a nucleus ${ }_{Z_{1}-1}^{A_{1}} \mathrm{X}$, which we will call nucleus $1^{\prime}$, and the nucleus ${ }_{Z_{2}}^{A_{2}} \mathrm{X}$, which will be called nucleus 2 , absorbs a $W^{+}$boson and turns into the nucleus ${ }_{Z_{2}+1}^{A_{2}} \mathrm{X}$, which will be called nucleus $2^{\prime}$. Thus, the filled circles in the diagram denote the matrix elements of the weak charged hadron current, $j_{\mu}^{(1)}$ and $j_{\rho}^{(2)}$. 
Similar to the time-dependent propagator of scalar particles in the momentum representation (4), the corresponding propagator of spinor particles can be easily found to be

$$
S_{i}^{\mathrm{c}}(p, T)=i \frac{\hat{p}+m_{i}}{2 p^{0}} e^{-i \frac{m_{i}^{2}-p^{2}}{2 p^{0}} T} .
$$

It is this expression that will be used for the calculations hereinafter.

Now we can write down the amplitude in the momentum representation corresponding to diagram (24) in the case, where the time difference $y^{0}-x^{0}$ between the events of production and detection is fixed and equal to $T$. Since the momentum transfer in both the production and detection processes is small, one can use the approximation of Fermi's interaction. Using the time-dependent fermion field propagator (25), where we retain the neutrino masses only in the exponential, we arrive at the amplitude summed over all the three neutrino mass eigenstates:

$$
\begin{aligned}
M= & -i \frac{G_{\mathrm{F}}^{2}}{4 p_{\mathrm{n}}^{0}} \sum_{i=1}^{3}\left|U_{1 i}\right|^{2} e^{-i \frac{m_{i}^{2}-p_{\mathrm{n}}^{2}}{2 p_{\mathrm{n}}^{0}} T} \times \\
& \times j_{\rho}^{(2)}\left(P^{(2)}, P^{\left(2^{\prime}\right)}\right) \bar{u}(k) \gamma^{\rho}\left(1-\gamma^{5}\right) \hat{p}_{\mathrm{n}} \gamma^{\mu}\left(1-\gamma^{5}\right) v(q) j_{\mu}^{(1)}\left(P^{(1)}, P^{\left(1^{\prime}\right)}\right),
\end{aligned}
$$

where $P^{(l)}$ and $P^{\left(l^{\prime}\right)}, l=1,2$, are the 4-momenta of the corresponding nuclei. Here and below we omit the fermion polarization indices for simplicity.

The squared modulus of the amplitude, averaged with respect to the polarizations of the incoming nuclei and summed over the polarizations of the outgoing particles and nuclei, factorizes in the approximation of massless neutrinos as follows:

$$
\begin{aligned}
\left\langle|M|^{2}\right\rangle & =\left\langle\left|M_{1}\right|^{2}\right\rangle\left\langle\left|M_{2}\right|^{2}\right\rangle \frac{1}{4\left(p_{\mathrm{n}}^{0}\right)^{2}}\left[1-4 \sum_{\substack{i, k=1 \\
i<k}}^{3}\left|U_{1 i}\right|^{2}\left|U_{1 k}\right|^{2} \sin ^{2}\left(\frac{m_{i}^{2}-m_{k}^{2}}{4 p_{\mathrm{n}}^{0}} T\right)\right], \\
\left\langle\left|M_{1}\right|^{2}\right\rangle & =4 G_{\mathrm{F}}^{2}\left(-g^{\mu \nu}\left(p_{\mathrm{n}} q\right)+\left(p_{\mathrm{n}}^{\mu} q^{v}+q^{\mu} p_{\mathrm{n}}^{v}\right)+i \varepsilon^{\mu v \alpha \beta} p_{\mathrm{n} \alpha} q_{\beta}\right) W_{\mu \nu}^{(1)}, \\
\left\langle\left|M_{2}\right|^{2}\right\rangle & =4 G_{\mathrm{F}}^{2}\left(-g^{\rho \sigma}\left(p_{\mathrm{n}} k\right)+\left(p_{\mathrm{n}}^{\rho} k^{\sigma}+k^{\rho} p_{\mathrm{n}}^{\sigma}\right)-i \varepsilon^{\rho \sigma \alpha \beta} p_{\mathrm{n} \alpha} k_{\beta}\right) W_{\rho \sigma}^{(2)},
\end{aligned}
$$

where the nuclear tensors $W_{\mu \nu}^{(1)}, W_{\rho \sigma}^{(2)}$ characterizing the interaction of nuclei 1 and 2 with the virtual $W$-bosons are defined as

$$
W_{\alpha \beta}^{(l)}=W_{\alpha \beta}^{(l, \mathrm{~S})}+i W_{\alpha \beta}^{(l, \mathrm{~A})}=\left\langle j_{\alpha}^{(l)}\left(j_{\beta}^{(l)}\right)^{+}\right\rangle, \quad l=1,2,
$$

their symmetrical parts $W_{\alpha \beta}^{(l, \mathrm{~S})}$ being real and the anti-symmetrical ones $i W_{\alpha \beta}^{(l, \mathrm{~A})}$ being imaginary.

Our next step is to find the differential probability of the process, where the intermediate neutrino momentum $p_{\mathrm{n}}$ is fixed by the experimental setting. Let us denote the 4-momentum $p: p^{2}=0$, the vector $\vec{p}$ satisfies the energy-momentum conservation in the production vertex and is directed from the source to the detector. According to the prescription formulated in Section 2 we multiply the squared modulus of the amplitude (27) by the delta function of energy-momentum conservation $(2 \pi)^{4} \delta\left(P^{(1)}+P^{(2)}-P^{\left(1^{\prime}\right)}-P^{\left(2^{\prime}\right)}-q-k\right)$, substitute $p$ instead of $p_{\mathrm{n}}$ everywhere in the amplitude and multiply the result by the delta function $2 \pi \delta\left(P^{(1)}-P^{\left(1^{\prime}\right)}-q-p\right)$, which fixes the virtual neutrino momentum, and integrate it over the phase volume of the final particles and nuclei. Besides this, now, when the virtual neutrino momentum is fixed, one can pass from the time interval $T$ to the distance travelled by 
the neutrino $L$ according to the formula $T=L p^{0} /|\vec{p}|$. Thus, we arrive at the differential probability, which also factorizes:

$$
\begin{aligned}
\frac{\mathrm{d}^{3} W}{\mathrm{~d}^{3} p}= & \left.\frac{1}{2 E^{(1)} 2 E^{(2)}} \int \frac{\mathrm{d}^{3} k}{(2 \pi)^{3} 2 k^{0}} \frac{\mathrm{d}^{3} q}{(2 \pi)^{3} 2 q^{0}} \frac{\mathrm{d}^{3} P^{\left(1^{\prime}\right)}}{(2 \pi)^{3} 2 E^{\left(1^{\prime}\right)}} \frac{\mathrm{d}^{3} P^{\left(2^{\prime}\right)}}{(2 \pi)^{3} 2 E^{\left(2^{\prime}\right)}}\left\langle|M|^{2}\right\rangle\right|_{\substack{p_{\mathrm{n}}=p \\
T=L p^{0} /|\vec{p}|}} \times \\
& \times(2 \pi)^{4} \delta\left(P^{(1)}+P^{(2)}-P^{\left(1^{\prime}\right)}-P^{\left(2^{\prime}\right)}-q-k\right) 2 \pi \delta\left(P^{(1)}-P^{\left(1^{\prime}\right)}-q-p\right)= \\
= & \frac{\mathrm{d}^{3} W_{1}}{\mathrm{~d}^{3} p} W_{2} P_{e e}(|\vec{p}|, L) .
\end{aligned}
$$

Here

$$
\frac{\mathrm{d}^{3} W_{1}}{\mathrm{~d}^{3} p}=\left.\frac{1}{2 E^{(1)}} \frac{1}{(2 \pi)^{3} 2 p^{0}} \int \frac{\mathrm{d}^{3} q}{(2 \pi)^{3} 2 q^{0}} \frac{\mathrm{d}^{3} P^{\left(1^{\prime}\right)}}{(2 \pi)^{3} 2 E^{\left(1^{\prime}\right)}}\left\langle\left|M_{1}\right|^{2}\right\rangle\right|_{p_{\mathrm{n}}=p}(2 \pi)^{4} \delta\left(P^{(1)}-P^{\left(1^{\prime}\right)}-q-p\right)
$$

is the differential probability of decay of nucleus 1 into nucleus $1^{\prime}$, a positron and a massless fermion with momentum $\vec{p}$, which can be viewed as an electron neutrino flavor state,

$$
W_{2}=\left.\frac{1}{2 E^{(2)} 2 p^{0}} \int \frac{\mathrm{d}^{3} k}{(2 \pi)^{3} 2 k^{0}} \frac{\mathrm{d}^{3} P^{\left(2^{\prime}\right)}}{(2 \pi)^{3} 2 E^{\left(2^{\prime}\right)}}\left\langle\left|M_{2}\right|^{2}\right\rangle\right|_{p_{\mathrm{n}}=p}(2 \pi)^{4} \delta\left(P^{(2)}+p-P^{\left(2^{\prime}\right)}-k\right)
$$

is the probability of interaction of a massless fermion, which again can be viewed as an electron neutrino flavor state, with momentum $\vec{p}$ and nucleus 2 with the production of nucleus $2^{\prime}$ and an electron, and we introduced a special notation

$$
P_{e e}(|\vec{p}|, L)=1-4 \sum_{\substack{i, k=1 \\ i<k}}^{3}\left|U_{1 i}\right|^{2}\left|U_{1 k}\right|^{2} \sin ^{2}\left(\frac{\Delta m_{i k}^{2}}{4|\vec{p}|} L\right)
$$

for the expression, which, in the standard approach, is called the distance-dependent electron neutrino survival probability. Thus, one finds that the differential probability of the whole process is the product of the differential probability $\mathrm{d}^{3} W_{1} / \mathrm{d}^{3} p$ of the production of a massless electron neutrino flavor state with a definite momentum, the probability $W_{2}$ of its interaction in the detector and the standard distance-dependent oscillating factor $P_{e e}(|\vec{p}|, L)$.

Finally we observe that the experimental situation fixes only the direction of the intermediate neutrino momentum, but not its length. However, the considered process of the neutrino production is a three-body decay, hence the neutrino momentum can have different lengths in a given direction. In order to take into account the neutrinos with all the possible momenta directed from the source to the detector, one has to integrate the differential probability (31) multiplied by $|\vec{p}|^{2}$ with respect to $|\vec{p}|$ from $|\vec{p}|_{\min }$ to $|\vec{p}|_{\max }$. In what follows, we assume nuclei 1 and 2 to be at rest and put their initial momenta $\vec{P}^{(1)}, \vec{P}^{(2)}$ equal to zero. Then the lower limit of integration determined by the threshold of the registration process and the upper one determined by the energy-momentum conservation in the production vertex are given by [23]

$$
|\vec{p}|_{\min }=\frac{\left(M_{\left(2^{\prime}\right)}+m_{e}\right)^{2}-M_{(2)}^{2}}{2 M_{(2)}}, \quad|\vec{p}|_{\max }=\frac{M_{(1)}^{2}-\left(M_{\left(1^{\prime}\right)}+m_{e}\right)^{2}}{2 M_{(1)}} .
$$

Here $M_{(\ldots)}$ are the masses of the corresponding nuclei and $m_{e}$ is the electron mass. As a result we arrive at the total probability of detecting an electron in the process under consideration:

$$
\frac{\mathrm{d} W}{\mathrm{~d} \Omega}=\int_{|\vec{p}|_{\min }}^{|\vec{p}|_{\max }} \frac{\mathrm{d}^{3} W}{\mathrm{~d}^{3} p}|\vec{p}|^{2} \mathrm{~d}|\vec{p}|=\int_{|\vec{p}|_{\min }}^{|\vec{p}|_{\max }} \frac{\mathrm{d}^{3} W_{1}}{\mathrm{~d}^{3} p} W_{2} P_{e e}(|\vec{p}|, L)|\vec{p}|^{2} \mathrm{~d}|\vec{p}| .
$$


As an example of application of this formula, we consider the process, where neutrinos are produced in the decay ${ }^{15} \mathrm{O} \rightarrow{ }^{15} \mathrm{~N}+e^{+}+v_{i}$ and detected in a Cl-Ar or Ga-Ge detector. Both the production and detection nuclear reactions belong to the so-called allowed transitions [24], and one can neglect the dependence of the nuclear form factors on the momentum transfer [24]. In this case the product of the production and detection probabilities can be approximated by the function

$$
\begin{aligned}
\frac{\mathrm{d}^{3} W_{1}}{\mathrm{~d}^{3} p} W_{2}= & C \sqrt{\left(|\vec{p}|_{\text {max }}-|\vec{p}|\right)\left(|\vec{p}|_{\text {max }}-|\vec{p}|+2 m_{e}\right)}\left(|\vec{p}|_{\text {max }}-|\vec{p}|+m_{e}\right) \times \\
& \times \sqrt{\left(|\vec{p}|-|\vec{p}|_{\text {min }}\right)\left(|\vec{p}|-|\vec{p}|_{\text {min }}+2 m_{e}\right)}\left(|\vec{p}|-|\vec{p}|_{\text {min }}+m_{e}\right) .
\end{aligned}
$$

We do not give the explicit expression for the normalization constant $C$, which is different for different production and registration processes. It is unimportant for us, since we will normalize the probability (36) to unity at the point $L=0$. Normalized distribution function (37) shows the relative contribution of the neutrinos with a given momentum to the probability of the whole process at $L=0$. The values of $|\vec{p}|_{\max }$ and $|\vec{p}|_{\min }$ for the processes under consideration are:

$$
|\vec{p}|_{\min }^{\mathrm{Ga}-\mathrm{Ge}}=232 \mathrm{keV}, \quad|\vec{p}|_{\min }^{\mathrm{Cl}-\mathrm{Ar}}=814 \mathrm{keV}, \quad|\vec{p}|_{\max }^{\mathrm{O}}=1732 \mathrm{keV}
$$

Normalized functions (37) for both $\mathrm{Cl}-\mathrm{Ar}$ and Ga-Ge detectors are shown in Figure 1.

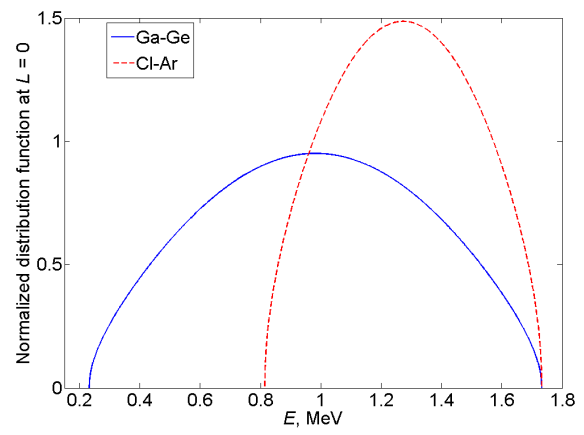

Figure 1. Normalized distribution functions (37) for an ${ }^{15} \mathrm{O}$ source and $\mathrm{Cl}-\mathrm{Ar}$ and $\mathrm{Ga}-\mathrm{Ge}$ detectors.

Of course, the approximation we use is rather rough. Nevertheless, it is sufficient to find the characteristic dependence of neutrino detection probability on the distance traveled. In so doing we use the following values of the neutrino masses and mixing angles [25]:

$$
\begin{gathered}
\Delta m_{21}^{2}=7.53 \cdot 10^{-5} \mathrm{eV}^{2}, \quad \Delta m_{32}^{2}=2.51 \cdot 10^{-3} \mathrm{eV}^{2}, \\
\theta_{12}=0.587, \quad \theta_{13}=0.146, \quad \theta_{23}=0.702 .
\end{gathered}
$$

Analytical integration in formula (36) with probability density (37) turned out to be impossible. Numerical integration gave the results presented in Figure 2 (the probability is normalized to its value at the point $L=0$ ). We see that the oscillation pattern depends on the detection process and the oscillations fade out with distance, which gives rise to a coherence length in our approach. This is due to the momentum distribution of the intermediate neutrinos. By analogy with interference in optics we introduce the visibility function:

$$
V(L)=\frac{I_{\max }-I_{\min }}{I_{\max }+I_{\min }}
$$




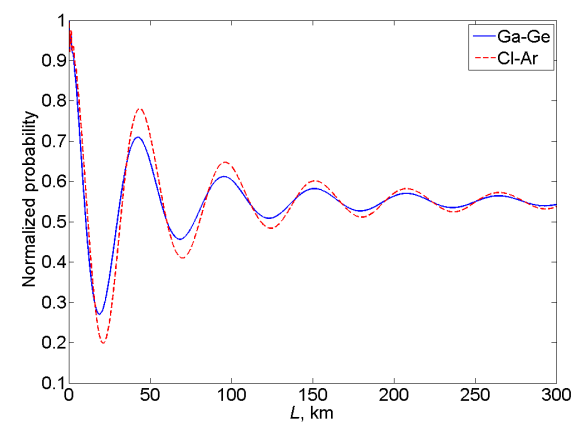

a) Distance $L$ from 0 to $300 \mathrm{~km}$.

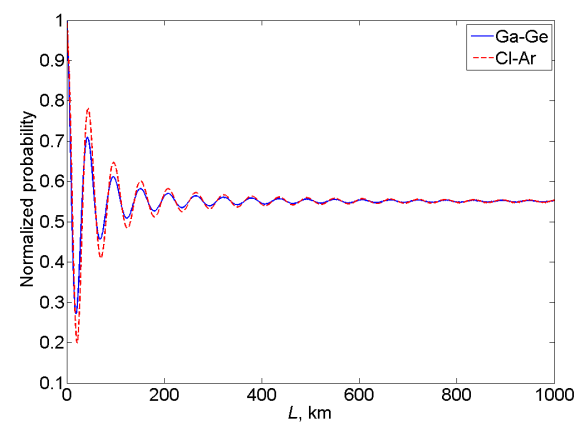

b) Distance $L$ from 0 to $1000 \mathrm{~km}$.

Figure 2. Normalized probabilities of the neutrino oscillation processes with the neutrino production in the ${ }^{15} \mathrm{O}$ decay and the registration by $\mathrm{Cl}-\mathrm{Ar}$ and $\mathrm{Ga}-\mathrm{Ge}$ detectors.

Here $I_{\max }, I_{\min }$ denote the relative neutrino registration probabilities in the adjacent maximum and minimum of the oscillation pattern. If we assume the condition of oscillation visibility to be $V(L)>0.1$, we arrive at the coherence lengths [17]

$$
L_{\mathrm{coh}}^{\mathrm{Ga}-\mathrm{Ge}} \approx 105 \mathrm{~km}, \quad L_{\mathrm{coh}}^{\mathrm{Cl}-\mathrm{Ar}} \approx 146 \mathrm{~km} .
$$

In the Ga-Ge case we have a wider momentum distribution than in the $\mathrm{Cl}-\mathrm{Ar}$ one, hence the Ga-Ge oscillation fade out more rapidly thus having a smaller coherence length.

\section{Conclusion}

A novel quantum field-theoretical approach to describing the processes passing at finite distances and finite time intervals is put forward. It is based on the Feynman diagram technique in the coordinate representation supplemented by modified rules for passing to the momentum representation, which take into account the geometry of neutrino oscillation experiments. It is shown that the approach allows one to consistently describe such processes as the displaced decay of an unstable particle, neutral kaon and neutrino oscillations, the results of the standard approaches being correctly reproduced. The approach makes use of only the particle mass eigenstates, while the flavor states and the states with definite strangeness are not needed. In the discussed approach neutrino oscillation is an interference process. By analogy with interference of non-monochromatic light, one can give a natural definition of the coherence length of neutrino oscillations, which, unlike the abstract definition of the standard approach, is tied to an experimental situation. The advantages of the approach are its extreme similarity to the standard Feynman diagram technique, the physical clearness and technical simplicity due to the use of plane waves only.

\section{Acknowledgments}

The authors are grateful to E. Boos, A. Lobanov, A. Pukhov, M. Smolyakov and Yu. Tchuvilsky for interesting and useful discussions. Analytical calculations of the amplitudes have been carried out with the help of the CompHEP and REDUCE packages. The work of V. Egorov was supported by the Foundation for the Advancement of Theoretical Physics and Mathematics "BASIS". 


\section{References}

[1] A. Pais and O. Piccioni, Phys. Rev. 100, 1487-1489 (1955)

[2] B.M. Pontecorvo, Sov. Phys. JETP 6, 429-431 (1957)

[3] V.N. Gribov and B.M. Pontecorvo, Phys. Lett. B 28, 493-496 (1969)

[4] R. Belušević, Springer Tracts Mod. Phys. 153, 1-182 (1999)

[5] C. Giunti and C.W. Kim, Fundamentals of Neutrino Physics and Astrophysics (Oxford University Press, UK, 2007)

[6] S. Bilenky, Lect. Notes Phys. 817, 1-255 (2010)

[7] K. Nakamura and S.T. Petcov, in: K.A. Olive et al. (Particle Data Group), Chin. Phys. C 38, 090001 (2014)

[8] C. Giunti et al., Phys. Rev. D 48, 4310-4317 (1993)

[9] W. Grimus and P. Stockinger, Phys. Rev. D 54, 3414-3419 (1996)

[10] M. Beuthe, Phys. Rept. 375, 105-218 (2003)

[11] A.G. Cohen, S.L. Glashow and Z. Ligeti, Phys. Lett. B 678, 191-196 (2009)

[12] A.E. Lobanov, Annals Phys. 403, 82-105 (2019)

[13] I.P. Volobuev, Int. J. Mod. Phys. A 33, 1850075 (2018)

[14] V.O. Egorov and I.P. Volobuev, Phys. Rev. D 97, 093002 (2018)

[15] I.P. Volobuev and V.O. Egorov, J. Exp. Theor. Phys. 128, 713-719 (2019)

[16] I.P. Volobuev and V.O. Egorov, Theor. Math. Phys. 199, 562-576 (2019)

[17] V.O. Egorov and I.P. Volobuev, Phys. Rev. D 100, 033004 (2019)

[18] N.N. Bogoliubov and D.V. Shirkov, Introduction to the theory of quantized fields (John Wiley \& Sons, 3d edition, New York, Chichester, Brisbane, Toronto, 1980)

[19] R.P. Feynman, Phys. Rev. 76, 749-759 (1949)

[20] R.P. Feynman, Phys. Rev. 76, 769-789 (1949)

[21] L.B. Okun, Leptons and Quarks (North Holland, New York, 1984)

[22] S.T. Petcov, Sov. J. Nucl. Phys. 25, 340 (1977)

[23] E. Byckling and K. Kajantie, Particle Kinematics (John Wiley \& Sons, London, New York, Sydney, Toronto, 1973)

[24] A. Bohr and B.R. Mottelson, Nuclear Structure: Volume I: Single-Particle Motion (World Scientific, New York, 1998)

[25] M. Tanabashi et al. [Particle Data Group], Phys. Rev. D 98, 030001 (2018) 